$\stackrel{W}{N}$

Global burnals Inc.

है ?

\title{
Effectiveness of Perineural Dexmedetomidine and Ropivacaine on Preventing Phantom Limb Pain in Patients Undergoing Femoral Supracondylar Amputation
}

By MD. Ramírez-Jaime, MD. Estrada-Sara \& MD. Martínez-Raquel

Abstract- Background: Phantom limb pain is a frequent complication after limb amputation, its pathophysiology is complex and includes changes in the peripheral nerve, dorsal root ganglia, spinal cord, cerebral cortex, and thalamus. At this time, there is not an effective drug for treatment and prevention of phantom limb pain. Perineural local anesthetic infiltration has obtained different results in the prevention of phantom limb pain, so we propose to combine an a2 agonist in peripheral nerve block to assess its effectiveness in preventing postoperative pain.

Keywords: phantom limb pain, analgesia, dexmedetomi-dine, periferial nerve block, supracondylar amputation.

GJMR-H Classification: NLMC Code: WE 300

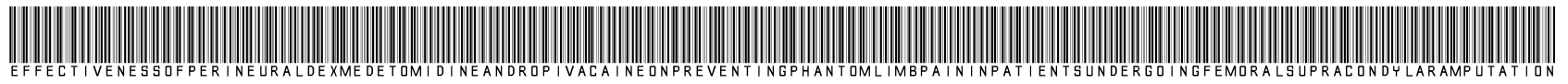

Strictly as per the compliance and regulations of:

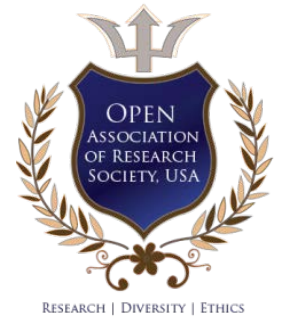

(C) 2020. MD. Ramírez-Jaime, MD. Estrada-Sara \& MD. Martínez-Raquel. This is a research/review paper, distributed under the terms of the Creative Commons Attribution-Noncommercial 3.0 Unported License http://creativecommons.org/licenses/by$\mathrm{nc} / 3.0 /$ ), permitting all non-commercial use, distribution, and reproduction in any medium, provided the original work is properly cited. 


\title{
Effectiveness of Perineural Dexmedetomidine and Ropivacaine on Preventing Phantom Limb Pain in Patients Undergoing Femoral Supracondylar Amputation
}

\author{
MD. Ramírez-Jaime ${ }^{\alpha}$, MD. Estrada-Sara $^{\circ} \&$ MD. Martínez-Raquel ${ }^{\circ}$
}

Abstract- Background: Phantom limb pain is a frequent complication after limb amputation, its pathophysiology is complex and includes changes in the peripheral nerve, dorsal root ganglia, spinal cord, cerebral cortex, and thalamus. At this time, there is not an effective drug for treatment and prevention of phantom limb pain. Perineural local anesthetic infiltration has obtained different results in the prevention of phantom limb pain, so we propose to combine an $\alpha 2$ agonist in peripheral nerve block to assess its effectiveness in preventing postoperative pain.

Methods: This was a prospective, double-blind, controlled, and simple randomized study. We included 30 patients undergoing surgery for supracondylar femoral amputation, older than 18 years, ASA I-III, in a sample calculation of $95 \%$ in Confidence Interval and $80 \%$ of statistical power. We included 15 patients per group managed with subarachnoid neuraxial block, and a perineural infiltration before the sciatical nerve section with $10 \mathrm{ml}$ of $0.5 \%$ ropivacaine $+50 \mathrm{mcg}$ dexmedetomidine (RD Group), or $0.5 \%$ ropivacaine $+0.9 \%$ saline solution (Group RS), in both groups We continued a perineural infusion with $0.15 \%$ ropivacaine using an elastomeric pump for 24 hours; We evaluated stump pain and phantom limb pain, upon admission to recovery, $8 \mathrm{hrs}, 24 \mathrm{hrs}$, 30 days and 3 months. The data analysis was carried out using the SPSS version 21 program; In all cases, we defined statistical significance when obtaining $p<0.05$.

Results: This study included a total of 30 patients, 15 patients in the RD group and 15 patients in the RS group. Statistical significance was found in the pain levels of the stump at rest at 24 hours, being lower in the RD group ( $p=0.003)$. This intervention also impacted the opioid requirements at 24 hours, requiring tramadol rescue $27 \%$ of the patients in the RD group, against $93 \%$ of the patients in the RS group $(p=0.0002)$. Regarding phantom limb pain, there was no incidence during the three months of follow-up in the RD group, against three patients who report mild phantom limb pain in the RS group.

Conclusions: The combination of ropivacaine with dexmedetomidine appears to be an effective intervention with minimal side effects to prevent the occurrence of acute and chronic pain as well as phantom limb pain in patients undergoing femoral supracondylar amputation as well as reducing the postoperative opioid requirements.

Author $\alpha \sigma \rho$ : Department of Anesthesiology of Guadalajara's Civil Hospital "Fray Antonio Alcalde". University of Guadalajara. Jalisco, México.e-mail: jarvil12@hotmail.com
Keywords: phantom limb pain, analgesia, dexmedetomidine, periferial nerve block, supracondylar amputation.

\section{INTRODUCTION}

$\longrightarrow$ atients undergoing limb amputation surgery are at risk of postoperative pain. Phantom limb pain (PLP) is a painful sensation referred to the absent limb after amputation; but it's not limited to the extremities, phantom pain has also been reported after loosing almost any body part, for example, nose, tongue or breast. ${ }^{1}$

The onset time of PLP varies from days to years, but it's common to be experienced between the first 24 hours to one week after amputation. ${ }^{1}$ In longterm studies, it's been observed a decrease in the frequency and duration of pain attacks over the years, which is accompanied by a decrement in its intensity, although in some cases, a worsening may occur. ${ }^{2,3,4}$

\section{a) Etiology and risk factors for PLP}

Up to date, the exact reason that some patients develop PLP and others do not is unknown. It has been observed that its development is multifactorial, involving from peripheral, central and also psychological mechanisms. ${ }^{5}$ There is not a clear relationship between age or sex and the appearance of PLP in the literature, and it's more likely to appear when it's a bilateral amputation and how proximal it is. ${ }^{6}$

There are studies about the influence of previous pain on an extremity as a factor for the development of PLP. ${ }^{6,7,8}$ There are reports that more than $50 \%$ of patients described PLP as similar to the pain they suffered before amputation. 9,10

At our country, type 2 Diabetes is the most common pathology causing amputation, referred as vascular diseases, it contributes to $81 \%$ of cases. On the other hand, traumatic amputations represent $16 \%$, tumors, and congenital diseases account for 3\%. ${ }^{11}$

b) Pathophysiology

We may assume that PLP is an example of neuropathic pain since it occurs mainly as a result of an attempt to reorganize the nervous system followed by 
the deference of all sensory information involving amputation with nerve section.

There are three mechanisms involved: "peripheral" given by the massive lesion discharge and the loss of nerve impulses towards the spinal cord. ${ }^{12}$ "spinal" Experimental studies have provided evidence of structural, physiological and neurochemical changes in the deferential neurons of the medullary dorsal horn. And it also causes alterations in "central" processing like transneuronal changes at the level of the nucleus of the thalamus. ${ }^{1,13}$

There are many pharmacological therapies to treat PLP once it appears, and some others are trying to prevent it, without consistent results that allow us to have a standardized treatment regimen. Madabhushi et al. Made a case report of a patient undergoing femoral supracondylar amputation, using bupivacaína (local anesthetic) and clonidine ( $\alpha 2$ agonist) with excellent results, the patient didn't report any pain in the stump and neither PLP 12 months after surgery. ${ }^{14}$ There are also systematic reviews about the use of dexmedetomidine ( $\alpha 2$ agonist) as an adjuvant to the local anesthetic for neuraxial and peripheral nerve blocks, to prolong the duration of analgesia compared to local anesthetic only. Cataloging Dexmedetomidine as a potential adjuvant and with more favorable pharmacodynamic and safety profile than Clonidine. ${ }^{15,16}$

Due the incidence of chronic pain or symptoms related to phantom limb, which may determine disability, a very poor quality of life by the dependence on consumption of opioid and non-opioid analgesics or neuropathic medications to decrease pain, we proposed preventive analgesia in this case, consisting in the application of an anesthetic and analgesic before the surgical incision of the sciatic nerve with the mainly aim to reduce the magnitude and duration of acute postoperative pain and so, assess the appearance or not of PLP, which it implies a decrease in morbidity and improvement in the quality of life; benefiting the patient, family and health institutions.

\section{il. Materials and Methods}

We carried out this study in Guadalajara's Civil Hospital "Fray Antonio Alcalde", México. Our study was a controlled, simple randomized, double-blind, clinical trial, conducted within a period of one year, from January 2018 to January 2019.

Inclusion criteria: Adult patients, scheduled for femoral supracondylar amputation, being due to traumatic cause, chronic disease and malignant tumor. Neuraxial block as anesthetic technique. American Society of Anesthesiologists's physical status I-III. With signed informed consent.

The exclusion criteria were: Refusal of the patient to participate, allergies known to any of the medications used, chronic hepatopathy disease, chronic renal disease, branch block or bradycardia and coagulation disorders.

Sample size calculation. We considered the total supracondylar amputations (110) performed at the Guadalajara's Civil Hospital during 2017, and established a significance level of $5 \%(0.05)$ and a statistical power of $80 \%$. We obtained a total of 30 patients (15 per group).

$R D$ Group. Perineural administration of $0.5 \%$ ropivacaine $10 \mathrm{ml}$ adding dexmedetomidine $50 \mathrm{mcg}$, and maintenance with perineural catheter infusion connected to an elastomeric pump with $0.15 \%$ ropivacaine for 24 hours.

RS Group. Perineural administration of $0.5 \%$ ropivacaine $10 \mathrm{ml}$ adding $0.5 \mathrm{ml}$ saline solution $0.9 \%$, and maintenance with perineural catheter infusion connected to an elastomeric pump with $0.15 \%$ ropivacaine for 24 hours.

Prior authorization by the Ethics and Research Committee of our Hospital, we assigned the patients in two groups randomly per sealed envelope.

Anxiolysis was given if required, with IV Midazolam $30 \mathrm{mcg} / \mathrm{kg}$. Antibiotic prophylaxis with Cephalothin $1 \mathrm{gr}$ IV. Preemptive analgesia with Paracetamol $1 \mathrm{gr}$ IV. Subarachnoid neuraxial block was administered, using hyperbaric bupivacaine $30 \mathrm{mcg} / \mathrm{kg}$ and intraoperative analgesia Parecoxib $40 \mathrm{mg}$ IV.

After dissection by the surgical team and before the section of the sciatic nerve, they identified the perineurium and infiltrated $10 \mathrm{ml}$ of $0.5 \%$ ropivacaine + 50 mcg dexmedetomidine (RD Group) or 0.5\% ropivacaine $+0.9 \%$ saline solution $(0.5 \mathrm{ml})$. We asked the surgeon to wait 5 minutes before performing the sciatic nerve section. After the amputation of the pelvic limb and before the surgical team closed by planes, a perineural catheter $(20 \mathrm{G})$ was placed and laterally exteriorized through a separate skin incision, connected to an elastomeric pump with $0.15 \%$ ropivacaine was placed in both groups for 24 hours.

We measured heart rate, blood pressure, sedation level (Ramsay Scale), at the end of the procedure, at PACU (Post-Anesthesia Care Unit), 8 hours, and up to 24 hours after. Also we recorded the pain in the stump, phantom pain using VAS (Visual Analogue Scale) in case of presenting at PACU, 8 hours, 24 hours, 30 days and up to 3 months after the surgery, all patients were followed by phone call after discharge from hospital. We recorded the rescue analgesia, and it was given with tramadol $1 \mathrm{mg} / \mathrm{kg}$ when VAS $>4$. We also monitored side and adverse effects associated with the use of dexmedetomidine such as bradycardia, hypotension, nausea, vomiting, and respiratory depression in PACU, 1 hour, 8, and 24 hours postoperatively.

We used the Statistical Package for the Social Sciences version $21^{17}$ for statistical analysis. 


\section{Results}

During January 2018 to January 2019, there were included 30 patients (15 per group) scheduled for supracondylar femoral amputation at our Hospital, under the anesthetic technique of subarachnoid block and perineural block of the sciatic nerve, which met the inclusion criteria from the study.

According to demographic data, of the 15 patients in the RD group were 2 women (13\%) and 13 men (87\%) with a mean age of 57.4 years (SD 20.7). We classified them according to physical status in ASA
I: 1 patient (7\%), ASA II: 1 patient (7\%), ASA 3: 13 patients (86\%), with the following preoperative diagnoses: diabetes mellitus $60 \%$, arterial occlusion $27 \%$, cancer $7 \%$, electric burn $7 \%$.

Of the 15 patients in the RS group, were seven women $(47 \%)$ and eight men (53\%) with a mean age of 60 years (SD 17.4). We classified them according to physical status in ASA I: 2 patients (13\%), ASA II: 1 patient (7\%), ASA 3: 12 patients $(80 \%)$, with the following diagnoses: diabetes mellitus 53\%, arterial occlusion 33 $\%$, cancer $7 \%$, fracture exposed $7 \%$. (Table 1 ).

Table 1: Demographic data

\begin{tabular}{|l|c|c|c|}
\hline Variable & $\begin{array}{c}\text { RD Group. Ropivacaína + Dexmedetomidina. } \\
\mathrm{N}=15\end{array}$ & $\begin{array}{c}\text { RS Group. Ropivacaína + Solución Salina. } \\
\mathrm{N}=15\end{array}$ & $\mathrm{P}(\mathrm{Cl}$ 95\%) \\
\hline & $($ Mean $\pm \mathrm{SD})$ & $($ Mean \pm SD) & \\
\hline Edad & $57.4 \pm 20.7$ & $60 \pm 17.4$ & 0.56 \\
\hline Género & & & \\
\hline Masculino & 13 & 7 & 0.83 \\
\hline Femenino & 2 & & \\
\hline ASA & 1 & 1 & \\
\hline I & 1 & 12 & \\
\hline II & 13 & & \\
\hline III & & & \\
\hline
\end{tabular}

Statistical significance when obtaining $P<0.05$. Abbreviations: $N$ (patients per group); ASA (Classification by the American Society of Anesthesiology); SD (Standard Deviation).

During the postoperative period, both groups were evaluated for pain intensity, both at rest and in movement, of the stump and phantom limb, from PACU, at 8 hours, 24 hours, 30 days, and up to 3 months.

Table 2: Pain Assessment (VAS) of the stump at rest and movement

\begin{tabular}{|c|c|c|c|c|}
\hline Variables & Evaluated Period & $\begin{array}{c}\text { RD Group } \\
\mathrm{N}=15\end{array}$ & $\begin{array}{c}\text { RS Group } \\
\mathrm{N}=15\end{array}$ & $\mathrm{p}$ (Cl 95\%) \\
\hline Pain Level & & (Main \pm SD) & (Main \pm SD) & \\
\hline Rest & PACU & 0 & $0.13 \pm 0.35$ & 0.143 \\
\hline & 8 hrs. & $0.93 \pm 0.59$ & $1.2 \pm 0.67$ & 0.426 \\
\hline & 24 hrs. & $0.73 \pm 0.59$ & $1.60 \pm 0.63$ & 0.003 \\
\hline & 30 days & 0 & $0.33 \pm 0.61$ & 0.099 \\
\hline & 3 months. & 0 & $0.06 \pm 0.25$ & 0.303 \\
\hline Movement & PACU & 0 & $0.20 \pm 0.41$ & 0.068 \\
\hline & 8 hrs. & $1.40 \pm 0.73$ & $1.60 \pm 0.73$ & 0.066 \\
\hline & 24 hrs. & $1.33 \pm 0.48$ & $1.73 \pm 0.45$ & 0.022 \\
\hline & 30 days. & 0 & $0.33 \pm 0.72$ & 0.189 \\
\hline & 3 months. & 0 & 0 & - \\
\hline
\end{tabular}

Statistical significance when obtaining $P<0.05$. Abbreviations: $N$ (patients per group); VAS (Visual Analog Scale); DS (Standard Deviation); PACU (PostAnesthetic Care Unit) hrs (hours). 


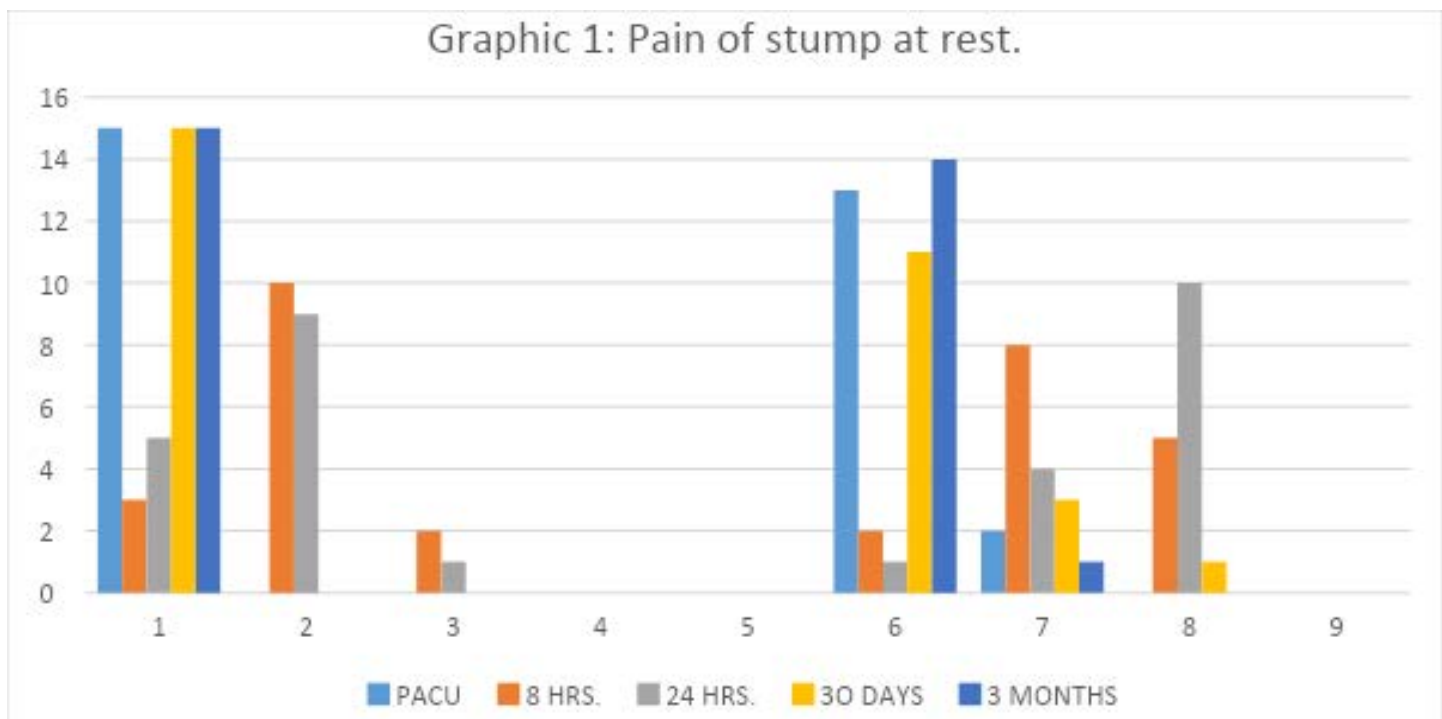

Abbreviations: RD (Ropivacaine + Dexmedetomidine), RS (Ropivacaine + Saline); PACU (PostAnesthetic Care Unit), Hrs (hours).

Pain values with VAS 4 and above, had the prescription of rescue with Tramadol at $1 \mathrm{mg} / \mathrm{kg}$. In the $\mathrm{RD}$ group, at 8 hours, five patients required Tramadol, at 24 hours four patients, and 48 hours 2 of them. In the RS group at 8 hours, nine patients were given tramadol

rescue, at 24 hours fourteen patients, and at 48 hours, 7 of them. Then it is possible to demonstrate with statistical significance that the perineural administration of dexmedetomidine does reduce the postoperative opioid requirements. (Table 3, Graph 2).

Table 3: Postoperative rescue with Tramadol

\begin{tabular}{|c|c|c|c|c|}
\hline Variables & Evaluated period & $\begin{array}{c}\text { RD Grup } \\
\mathrm{N}=15\end{array}$ & $\begin{array}{c}\text { RS Grup } \\
\mathrm{N}=15\end{array}$ & $\mathrm{p}$ (Cl 95\%) \\
\hline Tramadol rescue. & & Percent. & Percent. & \\
\hline & $8 \mathrm{hrs}$. & $33 \%$ & $60 \%$ & 0.143 \\
\hline & $24 \mathrm{hrs}$. & $27 \%$ & $93 \%$ & 0.0002 \\
\hline & $48 \mathrm{hrs}$. & $13 \%$ & $47 \%$ & 0.046 \\
\hline
\end{tabular}

Statistical significance when obtaining $P<0.05$. Abbreviations: hrs (hours).

Graphic 2. Postoperative Rescues with Tramadol.

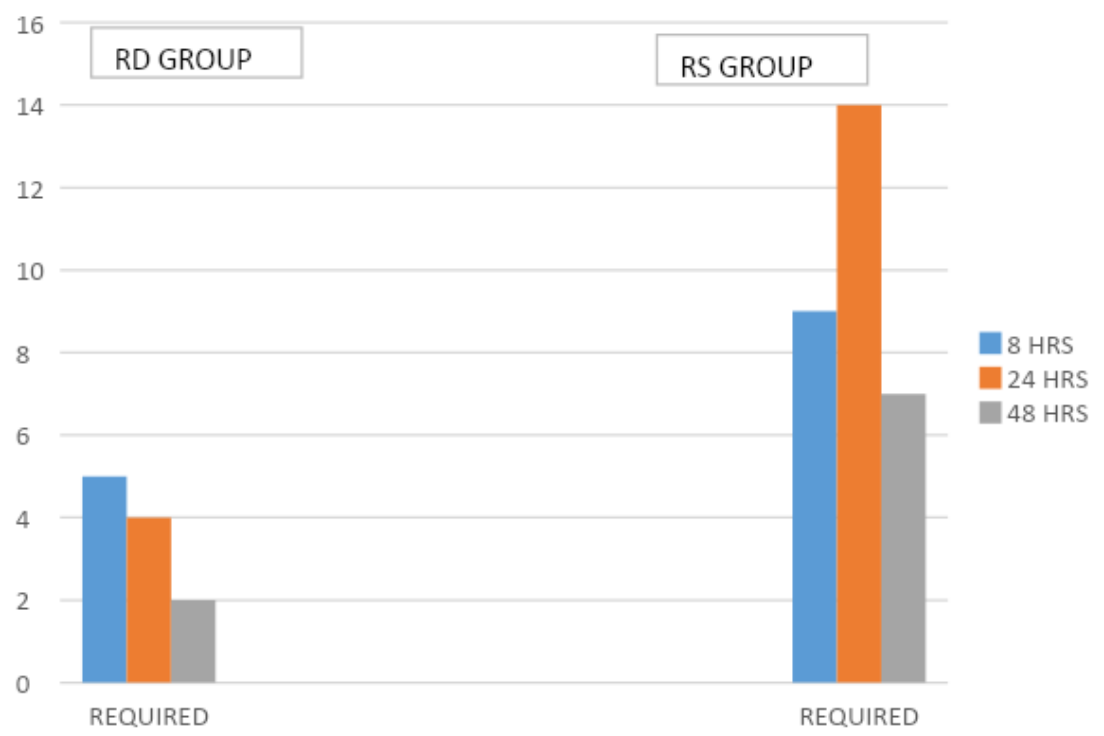

Abbreviations: $R D$ (Ropivacaine + Dexmedetomidine), RS (Ropivacaine + Saline Solution). 
We evaluated the appearance of phantom limb pain at rest and movement, which was not reported by any patient of the RD group from the moment of evaluation at PACU up to 3 months of follow-up. It is worth mentioning that two patients of the RD group presented prior amputation, one by contralateral first toe, and another ipsilateral transtibial, none reported previous phantom limb pain.
From the RS group, up to 30 days, one patient reported mild phantom limb pain and one more patient moderate resting pain; on movement three patients reported mild pain at 30 days and only one patient mild pain at 3 months, who we referred to the Hospital Pain Clinic for evaluation and treatment. Without being these statistically significant values. (Table 4)

Table 4: Assessment of phantom limb pain at rest and movement

\begin{tabular}{|c|c|c|c|c|}
\hline Variables & Evaluated period & $\begin{array}{c}\text { RD Group } \\
\mathrm{N}=15\end{array}$ & $\begin{array}{c}\text { RS Group } \\
\mathrm{N}=15\end{array}$ & $\mathrm{p}$ (Cl 95\%) \\
\hline Pain Score & & (Main \pm SD) & (Main \pm SD) & \\
\hline Rest & PACU & 0 & 0 & - \\
\hline & $8 \mathrm{hrs}$. & 0 & 0 & - \\
\hline & $24 \mathrm{hrs}$. & 0 & 0 & - \\
\hline & 30 days. & 0 & $0.20 \pm 0.56$ & 0.343 \\
\hline & Three Months. & 0 & $0.13 \pm 0.51$ & 0.309 \\
\hline Movement & PACU & 0 & 0 & - \\
\hline & $8 \mathrm{hrs}$. & 0 & 0 & - \\
\hline & 24 hrs. & 0 & 0 & - \\
\hline & 30 days. & 0 & $0.20 \pm 0.41$ & 0.068 \\
\hline & Three months. & 0 & $0.06 \pm 0.25$ & 0.309 \\
\hline
\end{tabular}

Statistical significance when obtaining $P<0.05$. Abbreviations: $N$ (patients per group); SD (Standard deviation), PACU (PostAnesthetic Care Unit) hrs (hours).

We decided to measure the possible side effects of perineurally administered medications on systemic blood pressure and heart rate in PACU, as well as the level of sedation (Ramsay scale) and oxygen saturation in PACU up to 24 hours after surgery.

We didn't find statistically significant differences in the values of systemic blood pressure, heart rate, and oxygen saturation after the intervention.
About sedation in the postoperative care unit, we found significant difference $(p<0.025)$ with a higher degree of sedation in the RD group with an average of 2.8 on the Ramsay scale. 2.4 in the RS group.

Table 5: Side effects on vitals and sedation after perineural block

\begin{tabular}{|l|c|c|c|c|}
\hline Variables & Evaluated Period & $\begin{array}{c}\text { RD Group } \\
\mathrm{N}=15 \\
\text { (Main } \pm \text { SD) }\end{array}$ & $\begin{array}{c}\text { RS Group } \\
\mathbf{N}=15 \\
\text { (Main } \pm \text { SD) }\end{array}$ & $p$ (Cl 95\%) \\
\hline Mean arterial pressure (mmHg) & PACU & $82.9 \pm 13.12$ & $83.5 \pm 12.04$ & 0.341 \\
\hline Heart rate (Bpm) & PACU & $75.2 \pm 10.56$ & $74.0 \pm 8.4$ & 0.555 \\
\hline Pulse-oximetry (\%) & PACU & $96.4 \pm 2.02$ & $95.7 \pm 1.90$ & 0.552 \\
\hline & $8 \mathrm{hrs}$. & $94.93 \pm 0.96$ & $95.33 \pm 0.72$ & 0.261 \\
\hline & $24 \mathrm{hrs}$. & $94.86 \pm 0.80$ & $95.06 \pm 0.59$ & 0.057 \\
\hline Sedation (Ramsay) & PACU & $2.80 \pm 0.41$ & $2.40 \pm 0.50$ & 0.025 \\
\hline & $8 \mathrm{hrs}$. & $2.20 \pm 0.41$ & $2.00 \pm 0.41$ & 0.068 \\
\hline & $24 \mathrm{hrs}$. & $2.00 \pm 0$ & $2.00 \pm 0$ & - \\
\hline
\end{tabular}

Statistical significance when obtaining $P<0.05$. Abbreviations: $N$ (patients per group); $S D(S t a n d a r d$ deviation), PACU (PostAnesthetic Care Unit) hrs (hours).

Of the possible adverse effects of the drugs used in our study, dizziness, nausea, vomiting, headache, and blurred vision were evaluated the first 24 hours after the perineural block. It was observed mainly in the RD group, dizziness in $13 \%$ at 8 hours, against $7 \%$ in the RS group $(p=0.068)$. Nausea in $13 \%$ at 8 hours in both groups. And headache $13 \%$ in the RD group against $20 \%$ in the RS group at 8 hours $(p=0.62$ ).
However, we didn't find statistically significant difference for any of the adverse effects measured. 


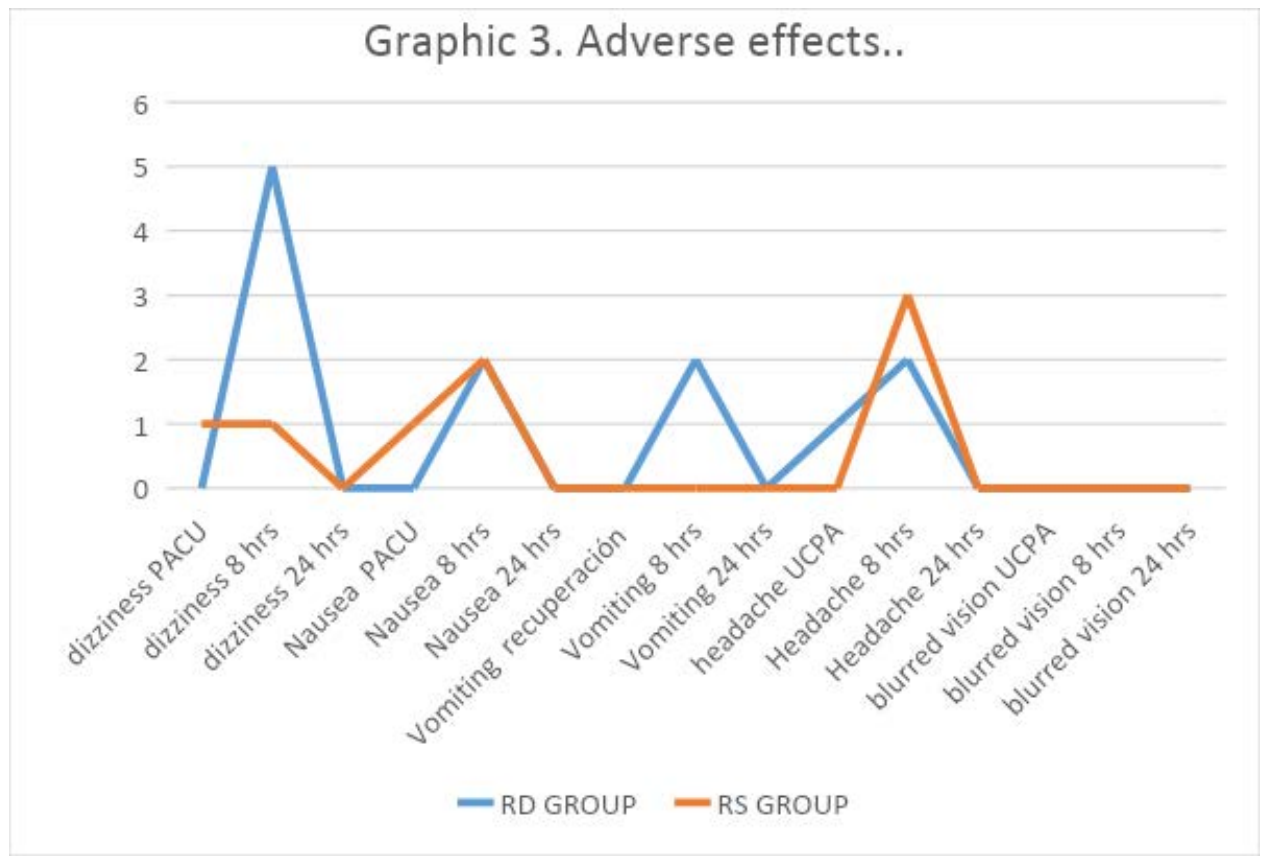

Abbreviations: RD (Ropivacaine + Dexmedetomidine), RS (Ropivacaine + Saline); HRS (hours).

\section{Discussion}

This study yielded significant data regarding the analgesic intervention of perineural infiltration with the combination of a local anesthetic (Ropivacaine) and an a2 agonist (Dexmedetomidine) since in the published literature there are only isolated reports of clinical cases of this combination, but no clinical trials and much less meta-analysis; there are some others of perineural infiltration with local anesthetic without additive for the prevention of PLP, and other about the addition of an a2 agonist for prolongation of peripheral nerve block, without assessing the impact on the prevention of phantom limb pain after amputation objectively.

Regarding the levels of postoperative pain, at 24 hours lower scores of pain at rest were found using the combination ropivacaine + dexmedetomidine $(p=$ 0.003); However, both interventions evaluated in this study with infiltration of local anesthetic and its postoperative infusion by using an elastomeric pump were effective for pain management, coinciding with the study by Borgh et al. In which they conclude that the use of a prolonged postoperative perineural perfusion of $0.5 \%$ ropivacaine is effective therapy for the treatment of phantom limb pain. ${ }^{18}$

Therefore, postoperative opioid consumption was also reduced as described by Bosanquet et al. in a systematic review and meta-analysis on the use of intraoperative placed perineural catheter with infusion of local postoperative anesthetic ${ }^{19}$. In this case, for our study, the addition of dexmedetomidine to perineural infiltration had a statistically significant difference in the reduction of the use of rescue tramadol at 24 hours, compared with the use of only local anesthetic $(p=0.0002)$.

\section{Conclusion}

Phantom limb pain, associated with the amputation of a member of the organism, is a frequent complication after each procedure, sometimes reported with very high incidence rates.

In the understanding of the pathophysiology for the development of this neuropathic pain, there were proposed different interventions for its treatment, but few focused on preventing its onset. One of those proposals is perineural infiltration and continuous infusion of local anesthetic adding an adjuvant to mitigate peripheral sensitization in nociceptors at the medullary level and central de-centralization (cortex and thalamus) in a preventive manner, avoiding the development of the PLP, which once it appears is very complex to treat.

In this study, we corroborate and verified the advantages of doing a preventive intervention as described with a local anesthetic but adding an a2 agonist, observing excellent results, and with no significant side effects.

Financial support and sponsorship

Nil.

Conflicts of interest

There are no conflicts of interest.

\section{References Références Referencias}

1. Luo $Y$, Anderson T. Phantom Limb Pain: A review. International Anesthesiology Clinics. 2016. 54, Number 2, 121-139. 
2. Jensen $\mathrm{T}$, Krebs B, Rasmussen $\mathrm{P}$. Inmediate and long-term phantom limb pain in amputee: incidente, clinical charasteristics and relationship to preamputation limb pain. Pain 1985; 21:267-78.

3. Dijkstra P, Geertzen H, Stewart R. Phantom pain and risk factors: A multivariate analisis. J of Pain and Sym Manag 2002; 24:578-585.

4. Ehde D, Czernieki J. Chronic pantom sensarions, phantom pain, residual limb pain and other regional pain after lower limb amputation. Arch Phys Med Rehabil. 2000;81:1039-1044.

5. Weeks SR, Anderson-Barnes VC, Tsao JW. Phantom limb pain: theories and therapies. Neurologist. 2010; 16:277-286.

6. Davis R. Pantom sensation, phantom pain and stump pain. Arch Phys Med Rehabil 1993; 74:79-91.

7. Nikolajsen L, Corner K. The influence of preamputation pain on postamputation stump and phantom pain. Pain 1997; 72:393-405.

8. Katz J, Melzack R. Pain memories in phantom limbs: review and clinical observations. Pain. 1990; 43:319-333.

9. Jensen $T$, Krebs B, Rasmussen $P$. Inmediate and long-term phantom limb pain in amputee: incidente, clinical charasteristics and relationship to preamputation limb pain. Pain 1985; 21:267-78.

10. Jensen $T$, Krebs $B$, Nielsen $J$, Rasmussen $P$. Phantom limb, phantom pain and stump pain in amputees during the first 6 month following limb amputation. Pain. 1983; 17:243-56.

11. Vázquez E. Academia Nacional de Medicina. Los amputados, un reto para el Estado. Acta de la sesión del 4 de marzo del 2015.

12. Weinstein S. Phantom pain. Oncology. 1994; 8:6570.

13. Nystrom B, Hagbarth KE. Microelectrode recordings from transected nerves in amputees with PLP. Neurosci Lett. 1981;27:211-216.

14. Madabhushi L, Reuben S. The efficacy of postoperative perineural infusion of bupivacaine and clonidine after lower extremity amputation in preventing phantom limb and stump pain. Journal of Clinical Anesthesia. 2007; 19, 226-229.

15. Abdallah F, Brull R. Facilitatory effects of perineural dexmedetomidine on neuraxial and peripheral nerve block: a systematic review and meta-analysis. British Journal of Anaesthesia. 2013. 110 (6): 91525.

16. El-Boghdadly, et. Al. Perineural dexmedetomidine is more effective than clonidine when added to local anesthetic for supraclavicular brachial plexus block: A systematic Review and meta-analysis. Regional Anesthesia and Acute Pain Medicine. 2017.124:6.

17. IBM Corp. Released 2012. IBM SPSS Statistics for Windows, Version 21.0. Armonk, NY: IBM Corp.

18. Borghi B, D'Addabbo M. The use of prolonged peripheral neural blockade after lower extremity amputation: the effect on symptoms associated with phantom limb syndrom. Rev Anesthesia and Analgesia. 2010; 111,5.

19. Bosanquet D, Glasbey J. Systematic review and meta-analysis of the efficacy of perineural local anaesthetic catheters after major lower limb amputation. Eur J Vasc Endovasc Surg. 2015; 50, 241-249. 